\title{
Kinematic Decoupled Core Paradigm: Counterrotation or Just Warping
}

\author{
Rubén J. Díaz ${ }^{1,2}$, Horacio Dottori ${ }^{3}$ \\ ${ }^{1}$ ICATE, CONICET, San Juan, Argentina \\ ${ }^{2}$ Gemini Observatory, La Serena, Chile \\ ${ }^{3}$ Instituto de Física, UFRGS, Porto Alegre, Brazil \\ Email: rdiaz@gemini.edu, dottori@if.ufrgs.br
}

Received 31 December 2015; accepted 17 June 2016; published 20 June 2016

Copyright (C) 2016 by authors and Scientific Research Publishing Inc.

This work is licensed under the Creative Commons Attribution International License (CC BY). http://creativecommons.org/licenses/by/4.0/

(c) (i) Open Access

\section{Abstract}

Several mechanisms have been proposed in recent years to explain kinematic decoupled cores (KDCs) in early type galaxies as well as the large differences in angular momentum between KDCs and host galaxy. Most of the proposed scenarios involve large fractions of merging events, high speed interactions with dwarf spheroidal galaxies, cusp effect of the dark matter density profiles, etc. We here argue that counterrotation as well as fast and slow rotation of disks or spheroids at the center of galaxies can also be explained by a misalignment of the central spheroid equatorial plane with regard to that defined by the observed external stellar rotation. Contrary to what happens at the outer region of disk galaxies, once instability has led to the inner warped core, the perturbed orbits can maintain a common orientation due to the rigid body like rotation at the central region of the galaxy. The spatial configuration that furnishes the smallest angular momentum difference between the KDC and the host galaxy is completely defined by observed parameters in the plane of the sky, namely, the inclination of the inner and outer disks and the angle between the two lines of nodes. As an example we modeled the paradigmatic and extreme case of the 2D radial velocity field of NGC 4382 nucleus. Tilt angles of the KDC not larger than 30 degrees also allow explaining fast and low rotators of the called "Sauron paradigm" in a unified scenario. The maximum for the three parameters, namely, velocity of the inner rotator, difference of position angle and difference with the outer rotation velocity of the whole Sauron sample, are consistently correlated in agreement with the proposed scenario. These quantities do not correlate with the galaxies magnitude, mass (since large and dwarf spheroidals show apparent counterrotation as well) or environment, also suggesting that an internal phenomenon like the central spheroid warping, that we are here proposing, may be at work.

\section{Keywords}

Galaxies Disks, Counterrotation, Inner Warps, Kinematics 


\section{Introduction}

More than thirty years ago [1] reported for the first time the discovery of a counterrotating core in the elliptical galaxy NGC 5813. Reference [2] reported not only that the core of the elliptical galaxy IC 1459 was counterrotating but also another challenging phenomenon, namely, that it was rotating about 4 times faster than the host galaxy. In the same year [3] had found that four out of seven slowly rotating large ellipticals presented rotating or counterrotating cores. Reference [3] also found a misalignment between the inner and the outer rotation axis in NGC 4765 and NGC 4406, concluding that in both galaxies the central core was decoupled from the main body. Studying the kinematics along the minor and major axis of 22 ellipticals [4] have found that $27 \%$ of them present a significant amount of minor axis rotation, what is expected if large ellipticals are tri-axial spheroids [5]. Reference [6] modeled numerically a merger between a large elliptical and a small companion, concluding that retrograde merging orbits might produce counterrotating cores, and that this type of core kinematical peculiarities can appear along the major, minor or intermediate axes.

A caveat of these models is that the smaller of the two merging galaxies has to have a core dense enough as to survive the tidal stripping and dynamical friction and to arrive hurtless to the very center of the main elliptical. Nevertheless [7] carried out HST +WFPC1 imagery of eight ellipticals with kinematically distinct core, arguing that the similarity in the central brightness distribution between these galaxies and "kinematically normal" ellipticals made it implausible a formation scenario in which small galaxies fell into big galaxies and dominated the light in the core region. Reference [8] have tackled this point through numerical simulations, showing that dissipationless accretion of a satellite is unlikely to form counter rotating cores in more than one percent of all ellipticals. The author claims that this low estimation results because even the densest satellites disrupt before reaching the large galaxy very center, forming a dense spherical core surrounded by a hot disk of particles of the large galaxy. Two new results further challenged the scenario of small satellites accretion to explain counter rotating cores in elliptical galaxies, namely, the discovery of kinematically distinct cores (KDCs, co- and counterrotating) in an increasing number of dwarf ellipticals with $M_{B} \geq-19$ magnitudes [9]-[12] and the striking similarity in the stellar content of the KDCs and the host galaxy [11] [13] [14]. Since the dwarf galaxies FS 76 and FS 373 reside in the groups of NGC 5044 and NGC 3258 respectively [9] propose that the slow co-rotating KDC in these galaxies might result from the harassment produced by the fly-by encounters with other galaxies. This scenario is valid to explain the counterrotating core of NGC 770 (satellite of NGC 772) [10] but not for explaining the origin of the counterrotating core of the nucleated galaxy VCC 510 in the Virgo cluster. With $M_{B} \cong$ -15.1 magnitudes it is the smallest dwarf known up to now to show a counterrotating core [12]. As mentioned by the authors, cases like VCC 510 should not be expected more than one encounter every 400 galaxies in a Hubble time.

There is not an unified scenario for the KDC phenomenon, but a consensus seems to exist about the existence of decoupled central structures cores, in scales running from ten of parsecs up to two kiloparsecs in a variety of galaxies with brightness between $-22<M_{B}<-16$ magnitudes. We explore in this letter a rather simple issue, namely, up to which extent projection effects produce a mirage leading to consider as different phenomena those that are strikingly similar. It is one of the best know effects in observational kinematics that it is not possible to disentangle how a disk galaxy rotates only by knowing which of the lines of the nodes extremes is receding, it is also necessary to know which is the minor axis nearest side. A spheroid whose equatorial plane is centrally warped will present differentiated projected rotation curves, depending on the two disks relative position with respect to the plane of the sky.

We explore in this letter all the observational consequences of taking this effect into account.

\section{Effects of Projection on Observed Kinematics of a Central Warped Disk}

The geometry and projected kinematics of a disk with warped external region have been extensively discussed in literature [15]-[17]. In the most common rotation curve case $\Omega \propto 1 / R$, slow and fast bending waves are produced on the warp [18] [19].

We will deal here with a rather different aspect of this phenomenon, namely, the very inner disk warping and its consequences on the disk observed kinematics. Such types of warping can survive for a long time in the region with rigid body like rotation curve. In this region the epicyclical and vertical frequencies are approximately constant $\kappa=v=\Omega_{0}=$ constant, then a bunch of orbits in a plane would preserve their identity (radial lines of particles remain aligned), eventually precessing. That makes a substantial difference with the external warps 
case. This property allows us to present the problem as that of a sharp warping which physically separates the central part of the equatorial plane of a rotating ellipsoidal galaxy, which we will simply call disk. Since heretofore in the Sauron paradigm literature no distinction has been made between disk galaxies (like NGC 7742, [20]) or galaxies with a strong spheroid (like NGC 474, [11]), we will in general refer to tilts or warps in the stellar rotation equatorial plane.

The line joining the two points of contact of the inner and outer disk will be called the line of warping and the angle between the planes of the outer and inner disk the angle of warping, $W$. The inclination of the outer and inner disk with respect to the plane of the sky is called $i_{o}$ and $i_{i}$ respectively.

\subsection{An Idealized Case: The Line of Warp Coincides with the Lines of the Nodes}

At first we will discuss an idealized but more didactic case in which the line of warping is along the line of the nodes, as shown in Figure 1. We have to distinguish two cases: $W<i_{o}$ and $W>i_{o}$.

In the case of $W<i_{0}$ and the inner and outer disk nearest halves are on the same side of the line of the nodes, when $i_{i}=i_{o}-W$ the inner disk will appear rotating slower than the outer disk (Figure 1(a)) (such would be the case of NGC 4203 rotation curve in Figure 2 [20]).

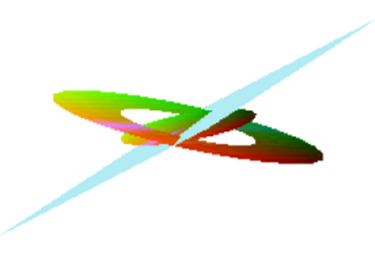

INNER SLOW ROTATOR

(a)

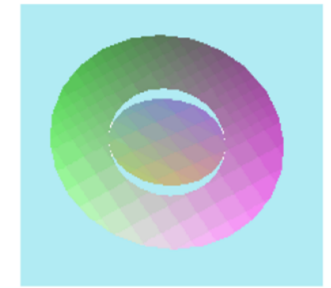

INNER FAST ROTATOR

(b)

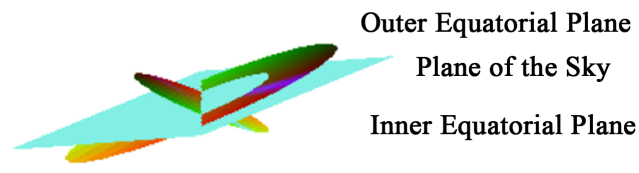

COUNTER ROTATOR

(c)

Figure 1. Blue surface represents the Plane of the Sky. Left, slow-rotator: inner disk less inclined than the outer disk with respect to the plane of the Sky. Center, fast-rotator: inner disk more inclined than outer disk with respect to the plane of the Sky. Right, counterrotator: on the same side of the Line of the Nodes inner and outer disk are located on different sides of the plane of the Sky.

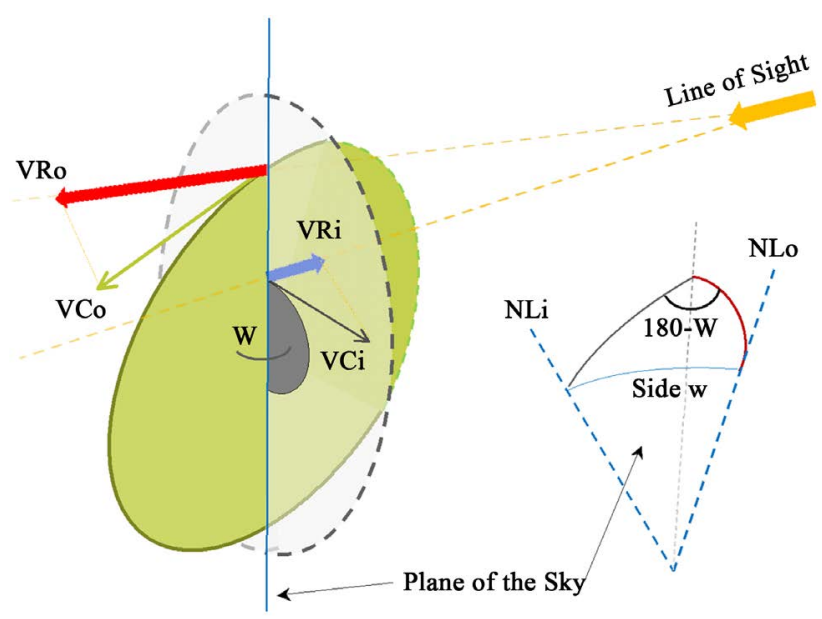

Figure 2. Schematic view of the counterrotation effect due to a warp affecting the relative orientation of the inner and outer equatorial planes of the rotating system. The Plane of the Sky is shown edge-on in the left panel and face-on in the right panel. We show in the left panel the angle of warping $\mathrm{W}$ as well as the circular velocity of inner $\left(V C_{i}\right)$ and outer $\left(V C_{o}\right)$ disk, as well as inner $\left(V R_{i}\right)$ and outer $\left(V R_{o}\right)$ disk observed radial velocities. In the right panel we show the line of the nodes of the inner $\left(N L_{i}\right)$ and outer $\left(N L_{o}\right)$ disks. We also show angle w, the angular separation between $N L_{i}$ and $N L_{o}, w=\left(N L_{i}-N L_{o}\right)$. Note that both inner and outer disk spin point in the same hemisphere, therefore any associated thick disk or spheroid will co-rotate. 
When $i_{i}=i_{o}+W$ and $W \leq 90^{\circ}$ we would see the inner disk rotating as a fast rotator (Figure 1 (b)), as would be the case of NGC 4526 [11].

If $W \geq i_{o}$ and $i_{i}=i_{o}+W \leq 90^{\circ}$ we will again have an inner fast rotator.

If $i_{i}=i_{o}-W$, the inner disk farthest part, behind the plane of the sky will correspond to the nearest part of the outer disk. Along the line of the nodes the inner disk receding side will coincide with the approaching side of the outer disk. In this case the inner disk will appear counterrotating to the outer disk (Figure 1(c)). A 2-D velocity map will show in this case an inner counter-rotating disk, with its major axis approximately aligned to that of the outer disk. Besides the Sauron examples, another galaxy presenting this behavior could be NGC 770 observed with GMOS + Gemini [10].

\subsection{The General Case}

The idealized situation, although didactic, is very hard to verify in real world. Indeed, a close inspection at SAURON 2-D velocity fields shows a variety of position angle difference of the inner and outer disks line of the nodes [11]. In this case we have to bear in mind that the lines of the nodes are the intersection of the plane of the disks and the plane of the sky. So we can construct a spherical triangle with center in the center of the galaxy in which the angles are $i_{o}, i_{i}$ and $W$ and the sides the angles between the two lines of the nodes and the intersection of both disks (Figure 2).

The inclination $\mathrm{i}_{0}$ of the external disk can be measured from images, as usually. 2-D velocity maps can provide the line of the nodes of the internal disk, its inclination $i_{i}$ as well as the angle between the two lines of the nodes $w$. In so doing we can determine the angle of warping:

$$
\cos W=\cos i_{o} \cdot \cos i_{i}+\sin i_{o} \cdot \sin i_{i} \cdot \cos w
$$

This allows us to recover the spatial disks location. Of course, this solution is not unique and leaves open the possibility of having real cases of counter rotating KDC.

\subsection{Two Extreme Cases}

The best source for examples is provided by the most complete sample of early type galaxies with 3D spectroscopic data, obtained with the Sauron instrument [21]. We developed a simple kinematic model for NGC 4382, which is one of the paradigmatic and extreme objects in the sample. This galaxy central region, first observed with Sauron [11] and later at higher resolution with OASIS, presents a counter rotating KDC with a small rotation amplitude $(30 \mathrm{~km} / \mathrm{s})$ [22]. The model radial velocity field was constructed with a Plummer spheroidal mass component fitted to the global observed radial velocity field up to $20^{\prime \prime}$, with a small inclination $\left(i_{0}=+10^{\circ}, P A_{o}=\right.$ $\left.90^{\circ}\right)$. Allowing for an abrupt but small change of inclination and line of nodes for radii smaller than $2 "\left(i_{\mathrm{i}}=-10^{\circ}\right.$, $P A_{i}=70^{\circ}$ ), we were able to reproduce the observed radial velocity field (Figure 3). We also show the case of NGC 770, whose parameters are quoted in the figure caption.

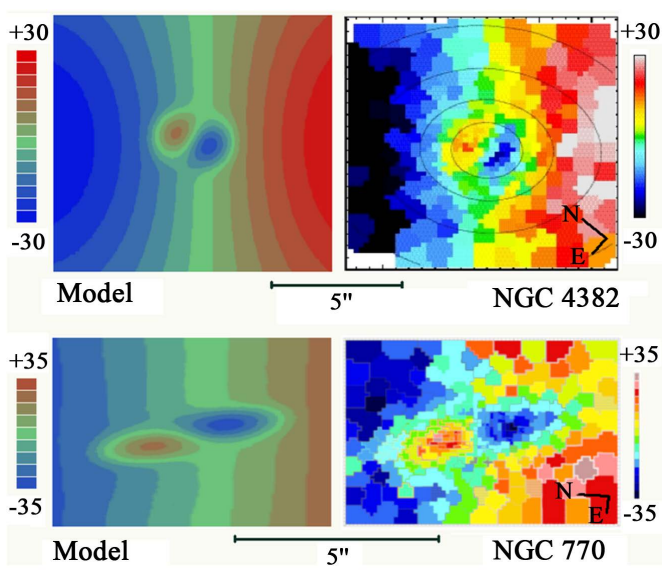

Figure 3. NGC 4382, see details in the text. For NGC 770 [10] our model suggest a large central warp with $i_{o}=-10^{\circ}, P A_{o}=0^{\circ}$ and for radii smaller than $2.5^{\prime \prime}, i_{i}=+60^{\circ}, P A_{i}=15^{\circ}$. A seeing of $0.7^{\prime \prime}$ has been assumed. Right panel shows the observed velocity field of both galaxies. 
Another clear example is constituted by the radial velocity field of the circumnuclear ring of gas in NGC 7742 [20] (Figure 4). These authors propose that the ring counter rotates respect to the stellar component inside the radius of the ring. Notwithstanding, this otherwise normal galaxy is face-on (the circumnuclear ring is indeed circular in the HST imagery) and the amplitude of the stellar velocities is modest. The gas rotation velocity has moderate values and even a slight tilt of $20^{\circ}$, of the gaseous ring (as in Figure 3, right) would explain the apparent counterrotation, without the need of a very complex and dynamically unstable configuration of the gaseous clouds rotating against the stellar streams in the galaxy equatorial plane.

\section{Characterizing the Warp Families}

We compiled the radial velocity fields of 28 early type disk and spheroidal galaxies [10] [20] [22]-[24]. We included those galaxies for which the inner rotation was apparent in the presented radial velocity field and we determined and compiled the position angles and radial velocity amplitude in the inner and outer spider diagrams, together with the host properties taken from [25] Tully (1988), including galaxy morphological classification, luminosity, HI content and local density of galaxies. The data table of the 28 objects studied is available on request to the authors.

As can be seen in Figure 5, the sample clearly separates in two halves at $50 \mathrm{~km} / \mathrm{s}$. The inner fast rotator family clearly correlates with the outer rotation velocity amplitude and, strikingly, there are no counterrotations over the $50 \mathrm{~km} / \mathrm{s}$ limit. This is fully consistent with the fact that a fast rotator projection can be generated by a small warp only when both the inner and outer disks near sides are on the same side of the line of nodes.

Within the same scenario, the correlation of maximum rotation velocities shown at the lower panel of Figure 4 is just in accordance with the fact that we are looking at the same rotation curve.

\section{Final Remarks}

Analysis of recently published 2D stellar kinematics of S0 and spheroidal galaxies yields to a series of concerns and questions:

- As has been previously reported, it is found that $30 \%$ of all Kinematically Decoupled Cores (KDCs) are counter rotating; can models account for such a large fraction of cores with a spin opposite to the global one?

- Statistical differences in orientations and radial velocity amplitude appear between counter rotating and prograde rotating cores; is there a unique family of KDCs in spheroidal galaxies?

- It has been reported that most of the KDCs do not show different colors or chemical properties respect to the host galaxy, nor it is apparent a difference between high and low density environments; can this result be concealed with the capture and merging hypothesis for KDCs?
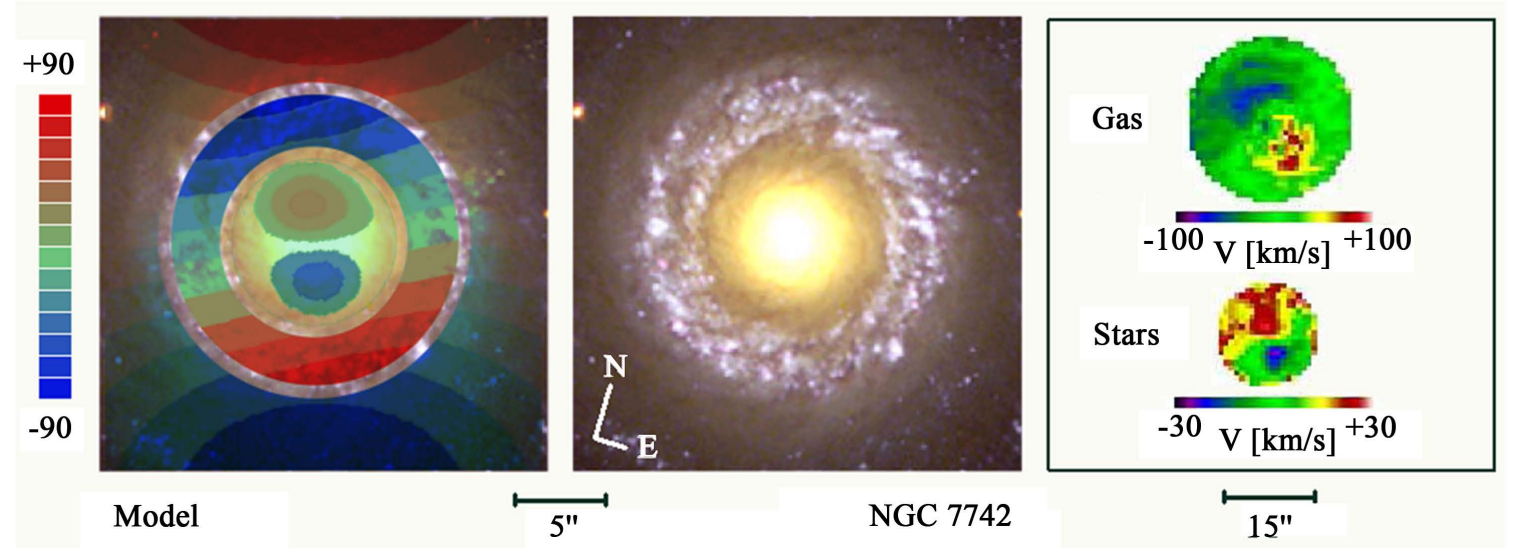

Figure 4. The most evident example of the proposed scenario: the well-known gaseous counterrotating circumnuclear ring [20] observed with Sauron at the WHT. Left: we propose a small tilt for the gaseous disk, $i_{q}=+10^{\circ}, P A_{q}=40^{\circ}$ and for the stellar velocity Field $i_{s}=-5^{\circ}, P A_{s}=30^{\circ}$, assuming a seeing of $1.5^{\prime \prime}$. Right: Hubble Heritage composed image of the galaxy. Note that the circumnuclear ring of star formation has an almost circular projection on the Sky plane, what suggest the faceon configuration. This case would correspond to the schematic view of Figure 1 (right) plus a small change of inner line of nodes, as shown in Figure 2. 


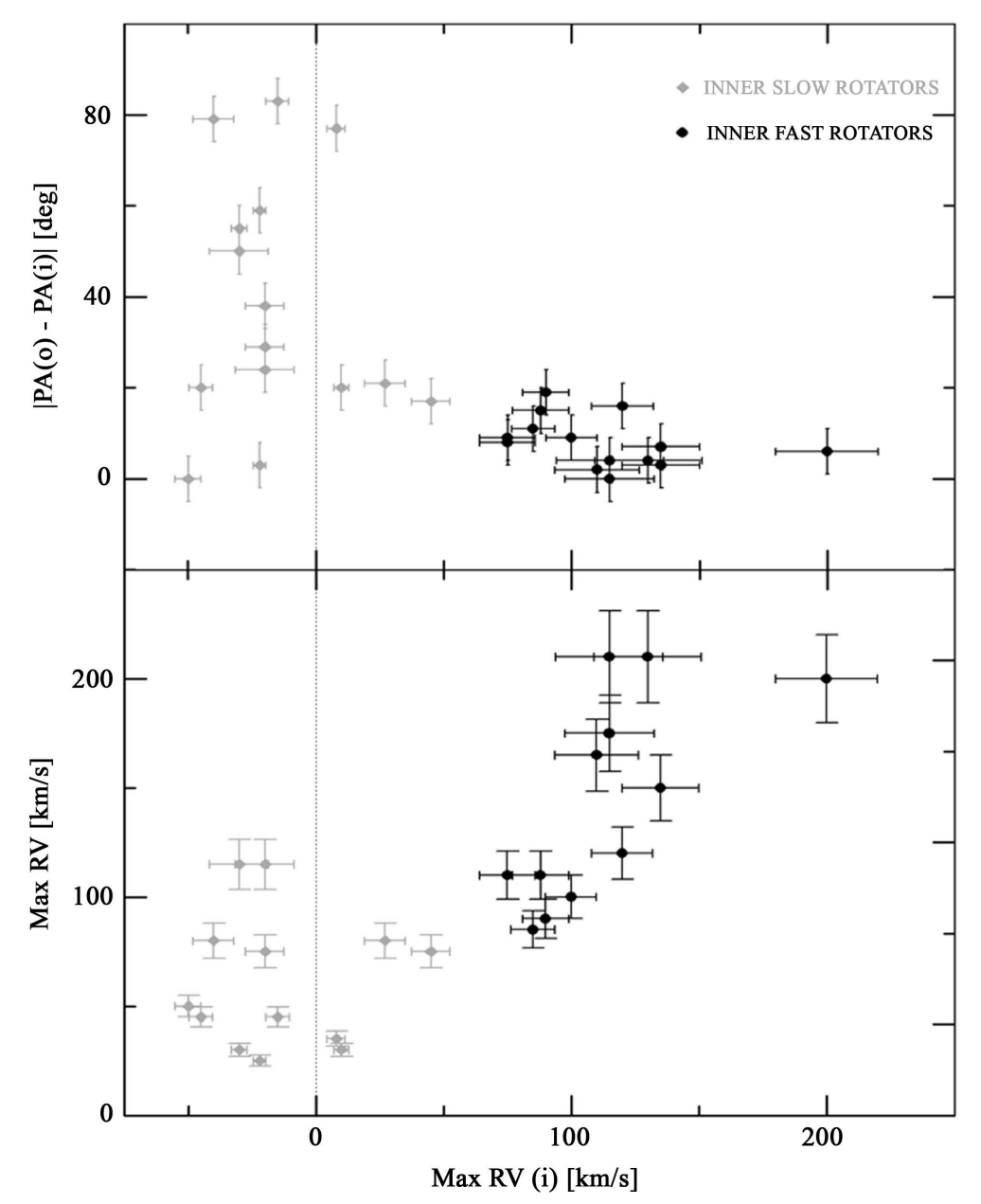

Figure 5. Upper panel: position angle difference $w=P A_{i}-P A_{o}$ vs. maximum velocity of the KDC. Lower panel: maximum velocity of the outer radial velocity field vs. Maximum velocity of the KDC. Note the slow-, counter- and fast-rotation families already noted in [22]. No other correlations have been found with intrinsic properties or the environment of the host.

- No large fractional differences (counter vs. direct) are apparent between the families of KDCs in low and high luminosity systems; can the same KDC formation scenario be applied to both dwarf and giant elliptical galaxies?

- It is also seen that $90 \%$ or more of the KDCs do not show a deep in the radial velocity dispersion maps and their velocity amplitudes are never larger than the global radial velocity amplitude at a few KPC radii; can they be considered as structures with more rotational support than the global host structure?

- It is apparent that "fast rotating" KDCs are located near to the equatorial plane of the global-host rotating system, and the counterrotation, which always shows a low radial velocity amplitude, can have any line of nodes orientation; can this be explained by a same scenario of subsystems' dynamical friction or even by a fly-by angular momentum interchange?

We have shown that a warp of equatorial plane at the center of the galaxy ellipsoid may easily reproduce paradigmatic cases of counterrotation. The spatial real disks' positions can be retrieved from observing parameters, namely the same that lead to interpret the same phenomena as counterrotation. The distribution of differences in position angle of inner and outer rings tends to agree better with a scenario of warps than with the mergers one. Warps include in a simpler way central fast-, slow- and counterrotation. The presence of KDC in a so wide variety of galaxy masses is also dynamically speaking less traumatic in the warp scenario than in the counter-rotation one.

Several scenarios have been proposed in the literature in the last ten years to explain KDCs and the apparent large differences in angular momentum. Notwithstanding we conclude that caution should be taken when consi- 
dering the impact of KDCs and counter rotating cores’ detections in galaxy formation and evolution scenarios, because a more precise evaluation of the observational and geometrical constrains is necessary in tackling this problem.

\section{Acknowledgements}

This paper was written while HD was visiting GEMINI-S. HD also acknowledges support from CNPq (Brazil). RD acknowledges support from CONICET through the research grant PIP-5697.

\section{References}

[1] Efstathiou, G., Ellis, R. and Carter, D. (1982) Further Observations of the Elliptical Galaxy NGC 5813A. Monthly Notices of the Royal Astronomical Society (MNRAS), 201, 975-990. http://dx.doi.org/10.1093/mnras/201.4.975

[2] Franx, M. and Illingworth, G. (1988) A Counter Rotating Core in IC 1459. Astrophysical Journal, 327, L55-L59. http://dx.doi.org/10.1086/185139

[3] Bender, R. (1988) Rotating and Counter-Rotating Cores in Elliptical Galaxies. A\&A, 202, L5-L8.

[4] Franx, M., Illingworth, G. and Heckman, T. (1989) Major and Minor Axis Kinematics of 22 Ellipticals. Astrophysical Journal, 344, 613-636. http://dx.doi.org/10.1086/167830

[5] Binney, J. (1985) Testing for Triaxiality with Kinematic Data. Monthly Notices of the Royal Astronomical Society (MNRAS), 212, 767-781. http://dx.doi.org/10.1093/mnras/212.4.767

[6] Balcells, M. and Quinn, P.J. (1990) The Formation of Couter Rotating Cores in Elliptical Galaxies. Astrophysical Journal, 361, 381-393. http://dx.doi.org/10.1086/169204

[7] Forbes, D., Franx, M. and Illingworth, G. (1995) Ellipticals with Kinematically Distinct Cores: WFPC1 Imaging of nearby Ellipticals. Astronomical Journal, 109, 1988-1995. http://dx.doi.org/10.1086/117425

[8] Bak, J. (2000) Retrograde Minor Mergers and Couter Rotating Cores. Ph. D. Thesis, DAI-B 61/06, Ohio University, Athens.

[9] De Rijcke, S., Dejonghe, H., Zeilinger, W. and Hau, G. (2004) Dwarf Elliptical Galaxies with Kinematically Decoupled Cores. Astronomy \& Astrophysics (A\&A), 426, 56-63. http://dx.doi.org/10.1051/0004-6361:20041205

[10] Geha, M., Guhathakurta, P. and van der Marel, R. (2005) NGC 770: A Counterrotating Core in a Dwarf Elliptical Galaxy. Astronomical Journal, 129, 2617-2627. http://dx.doi.org/10.1086/430188

[11] Emsellem, E., Cappellari, M., Peletier, R., McDermid, R.M., Bacon, R., et al. (2004) The SAURON Project-III: Integral-Field Absorption-Lines Kinematics of 48 Elliptical and Lenticular Galaxies. Monthly Notices of the Royal Astronomical Society (MNRAS), 352, 721-743. http://dx.doi.org/10.1111/j.1365-2966.2004.07948.x

[12] Thomas, D., Brimioulle, F., Bender, R., Hopp, U., Greggio, L., Maraston, C. and Saglia, R.P. (2006) A Counter-Rotating Core in the Dwarf Elliptical VCC 510. Astronomy \& Astrophysics (A\&A), 445, L19-L22. http://dx.doi.org/10.1051/0004-6361:200500215

[13] Carollo, C., Danziger, J., Rich, R.M. and Chen, X. (1997) Nuclear Properties of Kinematically Distinct Cores. Astrophysical Journal, 491, 545-560. http://dx.doi.org/10.1086/304979

[14] Davies, R., Kuntschner, H., Emsellem, E., Bacon, R., Bureau, M., Carollo, C.M., et al. (2001) Galaxy Mapping with the SAURON Integral-Field Spectrograph: The Star Formation History of NGC 4365. Astrophysical Journal, 548, L33-L36. http://dx.doi.org/10.1086/318930

[15] Rogstad, D., Lockhart, I. and Wright, M. (1974) Aperture-Synthesis Observations of HI in the Galaxy M83. Astrophysical Journal, 193, 309-319. http://dx.doi.org/10.1086/153164

[16] Rogstad, D., Lockhart, I. and Wright, M. (1976) Aperture-Synthesis Observations of HI in the Galaxy M33. Astrophysical Journal, 204, 703-711. http://dx.doi.org/10.1086/154219

[17] Rogstad, D., Crutcher, R. and Chu, K. (1979) Aperture-Synthesis Observations of HI in NGC 300. Astrophysical Journal, 229, 509-513. http://dx.doi.org/10.1086/156983

[18] Binney, J. and Tremaine, S. (1991) Galactic Dynamics. Princeton Univ. Press, Princeton, 539-548.

[19] Binney, J. (1985) Testing for Triaxiality with Kinematic Data. Monthly Notices of the Royal Astronomical Society (MNRAS), 212, 767-781.

[20] De Zeeuw, P.T., Bureau, M., Emsellem, E., Bacon, R., Carollo, C., Copin, Y., Davies, R., Kuntschner, H., Miller, B., Monnet, G., Peletier, R. and Verolme, E. (2002) The SAURON Project-II: Sample and Early Results. Monthly Notices of the Royal Astronomical Society (MNRAS), 329, 513-530. http://dx.doi.org/10.1046/j.1365-8711.2002.05059.x

[21] Bacon, R., Copin, Y., Monnet, G., Miller, B., Allington-Smith, J., Bureau, M., Carollo, C., Davies, R., Emsellem, E., 
Kuntschner, H., Peletier, R., Verolme, E. and de Zeeuw, P.T. (2001) The Sauron Project-I. The Panoramic Integral Field spectrograph. Monthly Notices of the Royal Astronomical Society (MNRAS), 326, 23-35. http://dx.doi.org/10.1046/j.1365-8711.2001.04612.x

[22] McDermid, R., Emsellem, E., Capellari, M., et al. (2004) OASIS High-Resolution Integral field Spectroscopy of the SAURON Ellipticals and Lenticulars. Astronomische Nachrichten, 325, 100-103. http://dx.doi.org/10.1002/asna.200310184

[23] Copin, Y., Cretton, N. and Emsellem, E. (2004) Axisymmetric dynamical models for SAURON and OASIS Observations of NGC3377. Astronomy \& Astrophysics (A\&A), 415, 889-903. http://dx.doi.org/10.1051/0004-6361:20034076

[24] Wernli, F., Emsellem, E. and Copin, Y. (2002) A 60 pc Counter-Rotating Core in NGC 4621. Astronomy \& Astrophysics (A\&A), 396, 73-81. http://dx.doi.org/10.1051/0004-6361:20021333

[25] Shields, J., Rix, H., McIntosh, D., Ho, L., Rudnick, G., Filippenko, A., Sargent, W. and Sarzi, M. (2000) Evidence for a Black Hole and Accretion Disk in the LINER NGC 4203. Astrophysical Journal, 534, L27-L30.

http://dx.doi.org/10.1086/312660 